\title{
Food parenting and child snacking: a systematic review
}

\author{
Rachel E. Blaine ${ }^{1 *}$, Alexandria Kachurak², Kirsten K. Davison ${ }^{3}$, Rachel Klabunde ${ }^{4}$ and Jennifer Orlet Fisher ${ }^{2}$
}

\begin{abstract}
Background: While the role of parenting in children's eating behaviors has been studied extensively, less attention has been given to its potential association with children's snacking habits. To address this gap, we conducted a systematic review to describe associations between food parenting and child snacking, or consuming energy dense foods/foods in between meals.

Methods: Six electronic databases were searched using standardized language to identify quantitative studies describing associations of general and feeding-specific parenting styles as well as food parenting practices with snacking behaviors of children aged 2-18 years. Eligible peer-reviewed journal articles published between 1980 and 2017 were included. Data were extracted using a standard protocol by three coders; all items were double coded to ensure consistency.
\end{abstract}

Results: Forty-seven studies met inclusion criteria. Few studies focused on general feeding $(n=3)$ or parenting styles $(n=10)$. Most studies focused on controlling food parenting practices $(n=39)$ that were not specific to snacking. Parental restriction of food was positively associated with child snack intake in 13/23 studies, while pressure to eat and monitoring yielded inconsistent results. Home availability of unhealthy foods was positively associated with snack intake in 10/11 studies. Findings related to positive parent behaviors (e.g. role modeling) were limited and yielded mixed results $(n=9)$. Snacking was often assessed using food frequency items and defined post-hoc based on nutritional characteristics (e.g. energy-dense, sugary foods, unhealthy, etc.). Timing was rarely included in the definition of a snack (i.e. chips eaten between meals vs. with lunch).

Conclusions: Restrictive feeding and home access to unhealthy foods were most consistently associated with snacking among young children. Research is needed to identify positive parenting behaviors around child snacking that may be used as targets for health promotion. Detailed definitions of snacking that address food type, context, and purpose are needed to advance findings within the field. We provide suggested standardized terminology for future research.

Keywords: Snacks, Feeding, Food parenting

\section{Background}

Childhood overweight and obesity persist as significant health risks for children globally $[1,2]$. Given that excessive energy intake is a primary driver for inappropriate weight gain among children, it is not surprising that child snacking has consistently increased in recent decades $[3,4]$. Snacking has been defined interchangeably in the literature as foods consumed between meals and/or

\footnotetext{
* Correspondence: rachel.blaine@csulb.edu

${ }^{1}$ Department of Family and Consumer Sciences, California State University, Long Beach, 1250 Bellflower Blvd, FCS FA-15, Long Beach, CA 90840-0501, USA

Full list of author information is available at the end of the article
}

consuming "snack foods", typically identified as energydense and nutrient-poor (i.e. candy, chips, cookies, sugary drinks). Individual study participants may also selfdefine snacking occasions. The inconsistency across definitions is problematic and limits the generalizability of findings. Snacking in between meals currently contributes an estimated one third of children's daily energy intakes in the United States [5] and a quarter of daily energy for youth in some European nations [6]. Though data on snacking and obesity in children are limited and equivocal, there is evidence that children who snack frequently consume greater energy, have 
poorer quality diets, and exhibit other risk factors for excessive weight gain $[7,8]$.

Although parental influence on children's overall eating behaviors and weight status has been studied extensively $[9,10]$, less attention has been given to how food parenting might affect the snacking behaviors of children. Food parenting includes both parent feeding practices, the specific behaviors or strategies that parents use to feed their children (i.e. pressuring a child to eat), and feeding styles, the generalized patterns of these practices. General parenting styles (e.g. uninvolved, authoritarian) approximate how caregivers engage with their children through interaction and disciplinary strategies and may also be informative in the context of child snacking, as different styles have been associated with a variety of childhood dietary and weight-related outcomes [11]. Current literature suggests that in order to promote healthy eating habits, parents must strike a balance between setting reasonable limits, providing healthful foods and structured eating occasions, and supporting children's unique food preferences and regulation of appetite $[12,13]$.

A recent theoretically guided conceptual model of snack-specific food parenting practices [14] identified four domains specific to snack feeding, which included Coercive Control, Permissiveness, Structure, and Autonomy Support. Coercive Control practices, such as restricting food or rewarding children with food, have been linked with increased energy intake, lower diet quality, and increased weight in children $[15,16]$. It is surmised that this domain may be particularly important in the context of snacking, as qualitative work suggests parents of young children, often use snack foods as tools to manage children's behaviors $[17,18]$. Permissive practices, such as feeding children to provide comfort, or having few rules or limits on snack intake, have been associated with excessive energy intake and elevated body mass index in children [19]. Given the low cost and portability of many processed snack foods, unrestricted access in the home may be especially problematic [20]. Conversely, it has been proposed that positive food parenting that provides Structure (e.g. routines, making healthy foods available) and Autonomy Support (e.g. role modeling, praise) is more likely to encourage children to establish healthy eating habits [21]. However, there are limited findings that describe such practices, and it is not clear what impact they may have on snacking intake among children [14]. Despite limited data, it is likely that overall parenting practices, whether positive or negative, have a differential impact on the quality of snack foods consumed by children.

To provide an overview of prominent findings in the literature, we conducted a systematic review to describe quantitative studies between 1980 and 2017 that have evaluated associations of parenting styles and food parenting practices with child snacking. Given the inconsistency in definitions, we describe all studies utilizing the word(s) snack/snacking, and provide distinctions between how they are measured and defined. We define snacking as consuming foods or beverages between meals, and snack foods are defined as energy-dense, nutrient poor foods/ beverages. Snacking behaviors refer to any behaviors related to snacking/consuming snack foods. To our knowledge, this is the first systematic review that assesses food parenting specifically in the context of child snacking. We are aware of one review that assessed the influence of two specific food parenting practices (e.g. parental pressure to eat and restriction) on children's dietary intake [22], but this review did not include a range of parenting behaviors and did not focus specifically on snacking.

The aims of this review were to: 1$)$ present characteristics of studies on parenting and child snacking, including study design, setting, participant demographics, and measures used to assess food parenting, 2) present the frequency with which food parenting practices were characterized in the literature, 3) summarize associations between food parenting practices and child snack intake, 4) describe characteristics of measures of child snacking, and 5) identify recommendations for future research.

\section{Methods}

\section{Search criteria}

To ensure consistency in data collection and presentation, we followed the Preferred Reporting Items for Systematic Reviews and Meta-Analyses (PRISMA) checklist to conduct our search [23] (Additional file 1) and registered our review with PROSPERO (Registration number: CRD42017062520). To standardize abstract review, we employed a protocol containing inclusion and exclusion criteria, along with an electronic search strategy for the study (Additional file 2).

We searched for English-language articles published in peer-reviewed journals in the following electronic databases: $\mathrm{CAB}$ Abstracts, Cumulative Index to Nursing and Allied Health Literature (CINAHL), Embase, PsycINFO, PubMed, and Web of Science. Key search terms were used to search titles, abstracts, and Medical Subject Headings and included text related to parents/caregivers (e.g. mother, father, parent), parenting style (e.g. parenting, parent-child relations, child rearing), food parenting (e.g. child feeding, control, restriction, pressure), and child snacking (e.g. snacks). Abstract files were downloaded, screened, assessed for eligibility, and organized by inclusion or exclusion in EndNote X7 by RB and AK. Full-texts of articles were assessed if they met all inclusion criteria.

\section{Eligibility criteria}

We included studies published between January 1980 and January 2017 in order to provide a scope of modern literature over the past four decades that reflects current parenting practices as well as those corresponding to increases in obesity prevalence in children over time [1]. 
Articles were included if they met the following criteria: 1) Measured snacking or snack-related behaviors of children aged $2-18$ years and 2) Measured the general parenting, feeding style, and/or food parenting practices of the child's parent or primary caregiver in the context of child snacking. We focused on children aged 2 and older to remove studies of infant breastfeeding and/or complementary feeding. We included studies with samples that included children younger than 2 only if solid food snacks were assessed (e.g. sample of toddlers and preschoolers aged 18 months - 5 years), but excluded studies with samples comprised of only children under 2 years.

Experimental studies that assessed children eating in the absence of hunger (EAH), following meals were included. Their protocols were developed to evaluate dimensions of satiety in children, but we believed the general paradigm was relevant because it focused on eating outside of meals [24]. More specifically, these studies evaluated the extent to which a meal suppressed subsequent intake of snack foods.

We excluded studies that did not directly assess primary caregivers (e.g. child care workers, laboratory feeding studies where parent was not present/assessed). We also excluded studies that did not appreciably measure food parenting, such as those solely assessing frequency of family meals or home availability of food (e.g. pantry audit), as these are often markers of other factors such as socioeconomic status.

We also excluded conference abstracts or dissertations because we sought to describe peer-reviewed journal articles. Qualitative studies and reviews were not included because they are not appropriate for drawing inferences about association. Articles were also excluded if their scope was outside the field of child/family nutrition (e.g. focus on oral health and dental caries) or only studied children with special healthcare needs (e.g. eating disorders, developmental delays) due to lack of applicability to the general population.

\section{Data extraction and analysis}

To ensure consistency all full-text articles were extracted and double coded by researchers (AK, RK, RB); 25\% were triple coded using the constant comparative method [25] to identify discrepancies in protocol interpretation and to reach a consensus when clarifying questions. Fewer than $5 \%$ of data items entered were in disagreement, and thus the protocol and data extraction tool were deemed appropriate for use.

Data extraction of full-texts occurred using a predefined list of items to be coded (Additional file 3) that were collected using Survey Gizmo for ease of data entry and summarization. After data extraction was complete, two researchers (AK and RK) also assessed study quality using existing tools: the National Institutes of Health Quality Assessment Tool for Observational Cohort and Cross-Sectional Studies [26] and the Quality Assessment Tool for Quantitative Studies designed to assess experimental studies [27]; $25 \%$ of studies were double coded to ensure study quality tool consistency and no disagreement was found. We used Stata/SE 12.1 (Stata Corporation, College Station, Texas, USA) to obtain frequencies for categorical variables and mean values and standard deviations for continuous variables.

\section{Study characteristics}

We documented general study information such as publication date, country, journal name, and study design. To describe study samples, we assessed age of target children, populations recruited (e.g. low-income, minority), and sample sizes of caregivers/children. To describe participant demographics, we examined caregiver race/ethnicity, gender (i.e. mothers vs. fathers), and level of education.

We described the extent to which studies reported on important demographic information associated with child feeding (e.g. parent education, race/ethnicity, inclusion of male caregivers) as well as instrument quality to see how often validated tools were used in their intended way (e.g. all items vs. select subscales vs. individual items), and the level of dietary assessment (e.g. 24-h recall vs. food frequency questionnaire) [9, 28]. Additionally, we described whether or not child snacking outcomes were predefined by the researchers before the outset of data collection, or defined post-hoc during analysis. We also examined the sample sizes and journals of publication to provide a general discussion about the diversity in publication. Finally, we described quality ratings for cohort and crosssectional studies (Range: Good, Fair, Poor) and experimental studies (Range: Strong, Moderate, Weak) using existing tools $[26,27]$.

\section{Measures of food parenting}

We collected data on whether general parenting style vs. specific practices were assessed and whether measures were snacking-specific. We also identified the type of practices studied using a pre-determined list of specific snack-feeding practices (e.g. role modeling, rewarding behavior) based upon a recently published conceptual model of food parenting practices specific to child snacking [14]. Practices were organized by four higher dimensions from the conceptual model: Coercive Control, Structure, Autonomy Support, and Permissiveness.

\section{Association between food parenting and child snacking}

We summarized study results on the association between food parenting and child snacking outcomes. We postcoded these result summaries as positive, negative, null, or 
mixed in order to summarize trends in association. Since both the exposure (food parenting) and outcome (child snacking) were measured in myriad ways and not generalizable quantitatively, we opted to conduct a narrative summary of our findings using tables and figures at the level of each individual study.

\section{Measures of child snacking}

We examined the types of measures used to assess child snacking, and collected data on the source (i.e. parent vs. child), use of validated tools, how "snack" was defined in both the tools and in the analysis post-hoc, and what types of contextual information was presented about child snacking (e.g. timing, nutrient profile, frequency).

\section{Results}

\section{Study characteristics}

Our search yielded 2846 articles, of which 84 duplicates were identified and removed (Fig. 1). After reviewing 2762 abstracts based upon inclusion and exclusion criteria (Table 1), 2696 were excluded and 66 were included for full-text assessment. Of full texts reviewed, 47 were included for analysis [13, 18, 29-73]. The primary reason for exclusions was that parenting/feeding practices were not assessed.

We present a brief narrative description of each study, the measures used, and study quality in Table 2 and a summary of overall study characteristics in Table 3 . Nearly half of all studies $(n=31)$ were published within the past 5 years. More than $90 \%$ of all studies occurred in four Western nations: the United States $(n=14,29.8 \%)$, the Netherlands $(n=12,25.5 \%)$, Australia $(n=8,17.0 \%)$, and the United Kingdom ( $\mathrm{n}=8,17.0 \%)$. With the exception of Appetite, which published 36\% of eligible articles, studies were published in a variety of journals $(n=25)$, with most journals publishing 1-2 studies each. There was significant diversity in authorship as well, with no author contributing more than 3 studies to the literature.

The majority of studies were cross-sectional $(72.3 \%$, $n=34)$, followed by longitudinal $(12.8 \%, n=6)$, and experimental $(14.9 \%, n=7)$. A unique grouping of experimental studies focused on EAH $(n=6)$. Most studies consisted of caregivers only $(n=15,31.9 \%)$ or caregiverchild dyads $(n=21,44.7 \%)$, compared with those recruiting children who self-reported on caregivers' practices $(n=11,23.4 \%)$. The mean sample size of participants or caregiver-child dyads was $n=693$ (standard deviation: 789, range: 35-2814, median: 377). Most studies focused on elementary-aged children $(n=30,63.8 \%)$. About $40 \%$ of studies $(n=20)$ reported on race/ethnicity of caregivers. While the majority of samples were predominantly white, a third of studies included samples that were predominantly non-white $(\mathrm{n}=6)$.
Overall, quality was high across cross-sectional and observational articles, with 39/40 receiving a Good quality rating (Range: Good, Fair, Poor) (Table 2). Among experimental studies $(n=6)$ quality was weaker due to a lack of reporting study participation rates (Range: Strong, Moderate, Weak); most experimental studies scored as moderate $(n=4)$ compared with weak $(n=1)$ or strong $(\mathrm{n}=1)$.

Most studies defined the gender of caregivers $(n=29$, $55.3 \%$ ) who were predominantly female. Forty percent of studies exclusively contained mothers/female caregivers ( $n=12)$; when included, males made up $11 \%$ of caregiver samples on average. Although these studies distinguished between male and female caregivers, only about one third $(n=10)$ explicitly mentioned the word "father" or defined the number of fathers in their sample. Most studies reported caregiver level of education $(n=34$, $72.3 \%$ ), with two studies reporting that their samples contained at least $40 \%$ of caregivers with a low level of education.

\section{Measures of food parenting}

The most commonly used tool adapted to measure food parenting practices was the Child Feeding Questionnaire ( $n=16,34.0 \%$ ) [74], followed by the Comprehensive Feeding Practices Questionnaire $(\mathrm{n}=3)$ [75]. General feeding styles $(n=3)$ or parenting styles $(n=10)$ were examined in fewer studies than specific food parenting practices $(n=42)$, and often focused their findings on specific practices within styles; few studies evaluated parenting specific to child snacking $(\mathrm{n}=10)$.

Using a theoretically-driven conceptual framework [14], we summarized the frequency with which specific food parenting practices were described across four dimensions of snack feeding in Fig. 2. The practices are presented across the four key dimensions (Coercive Control, Structure, Autonomy Support, and Permissiveness), to indicate how many studies provided data about each practice. Studies appeared to focus on more negative aspects of food parenting, with a strong focus on the dimension of coercive control $(n=39,90.0 \%)$ in the context of child snacking. Within this dimension, specific behaviors related to restriction $(n=32)$ and pressure to eat $(n=20)$ were most often described. Within the dimension of structure $(\mathrm{n}=32,68.0 \%)$, most studies measured home availability of healthy foods $(n=25)$ and monitoring of food intake ( $n=17)$, compared with fewer studies examining planning and routines $(n=8)$ and home availability of healthy foods/snacks $(n=12)$. Fewer studies described practices within the dimension of autonomy support $(n=20$, 42.5\%) and permissiveness $(n=15,31.9 \%)$, where home availability of unhealthy food $(\mathrm{n}=12)$ was assessed most frequently. 


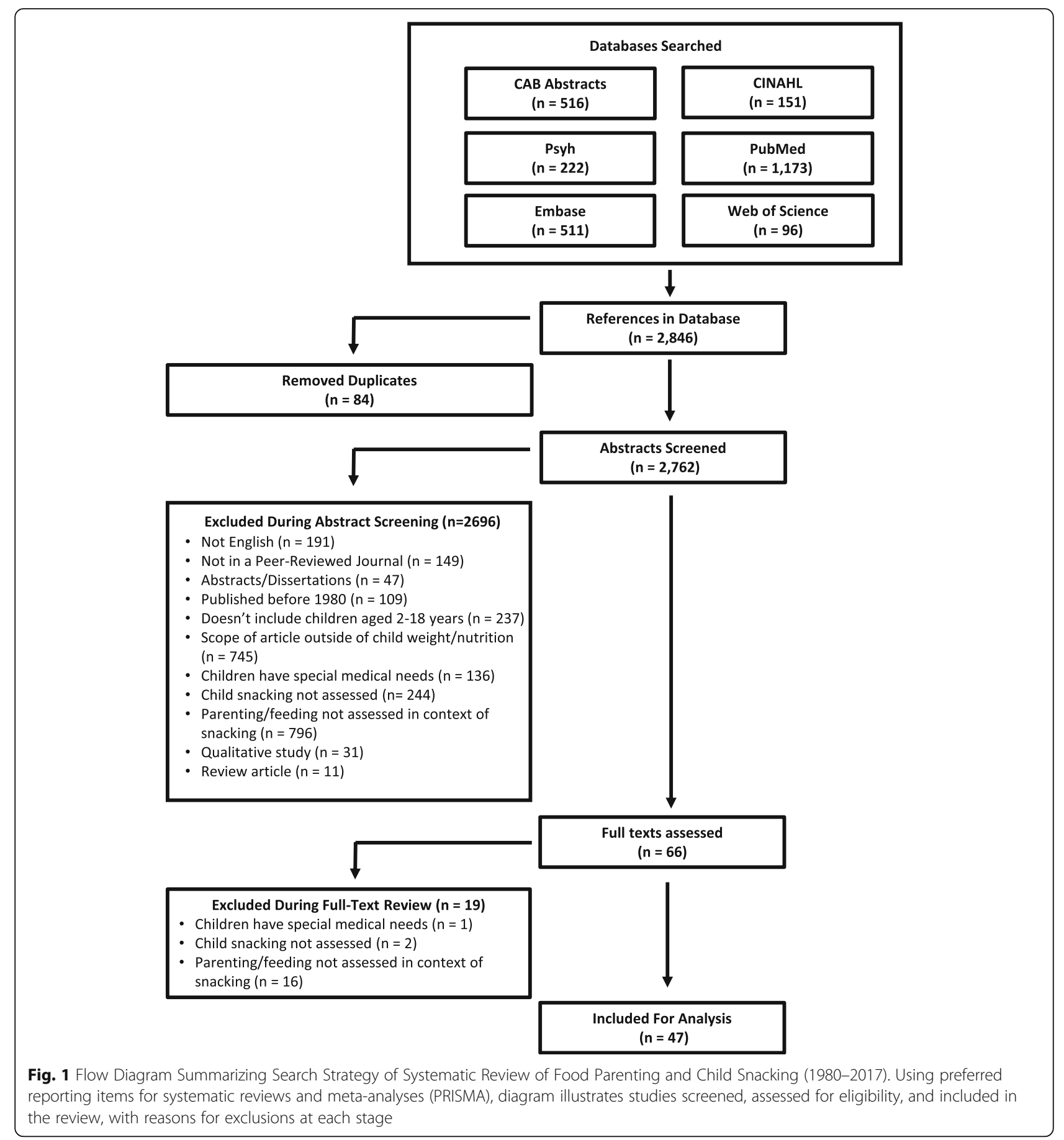

\section{Association between food parenting on child snacking}

We summarize associations of the most commonly studied aspects of parenting with child snacking in Fig. 3. No noticeable differences in trends based on feeding practices versus feeding or parenting styles were observed. Parental restriction of food was positively associated with child snack intake in 13/23 studies ( $\mathrm{n}=2$ experimental, $\mathrm{n}=2$ longitudinal, $n=9$ cross-sectional), while pressure to eat and monitoring yielded inconsistent results. Home availability of unhealthy foods was positively associated with snack intake in $10 / 11$ studies $(n=8$ cross-sectional, 2 = experimental). Instrumental feeding was described in 7 studies and was typically a combination of coercive controlling practices (e.g. restriction and rewarding with food). Findings related to positive parent behaviors (e.g. role modeling, reasonable rules about eating) were limited to less than a fifth of all studies $(n=9)$. Four of seven studies found parent food rules were negatively associated 
Table 1 Inclusion and Exclusion Criteria

\begin{tabular}{|c|c|}
\hline Inclusion Criteria & Exclusion Criteria \\
\hline $\begin{array}{l}\text { 1. Measured snacking or snack-related behaviors of children } \\
\text { aged } 2 \text { years to } 18 \text { years through either objective (e.g., } \\
\text { meal observations) or subjective (i.e., self-report) methods. } \\
\text { This could include nutrient intake, snack foods, frequency, } \\
\text { quality, or context. } \\
\text { 2. Measured the feeding style, feeding practices, and/or } \\
\text { parenting style of the child's parent or primary } \\
\text { caregiver through self-report of caregiver, child, or } \\
\text { direct observation (e.g. observed snack time) in the } \\
\text { context of child snacking. }\end{array}$ & $\begin{array}{l}\text { 1. Not in English } \\
\text { 2. Published prior to } 1980 \\
\text { 3. Not in a peer-reviewed journal (e.g. TIME magazine) } \\
\text { 4. Not a research article (e.g. published in Pediatrics but is an interest piece } \\
\text { or compilation of abstracts) } \\
\text { 5. Scope of article is outside of child/family nutrition or weight status (e.g. } \\
\text { focus on oral health, a particular foodstuff, etc.) } \\
\text { 6. Population studied was not children ages 2-18. As long as child was under } \\
\text { 18 at baseline, we can use the study. } \\
\text { 7. Exclude studies of nursing } \\
\text { 8. Population focused on children with special healthcare needs (e.g. feeding } \\
\text { disorders, diabetes, eating disorders } \\
\text { 9. Child snacks or snacking not assessed } \\
\text { 10. Parenting/parent feeding and child snacking not examined together } \\
\text { 11. Review paper } \\
\text { 12. Qualitative paper }\end{array}$ \\
\hline
\end{tabular}

${ }^{a}$ Did not include family meals or parent diet as a marker of food parenting

${ }^{b}$ Excluded if primary caregivers were not assessed at all (e.g. a study of the feeding patterns of child care workers)

with snack intake. Based on the small sample sizes, it is not possible to identify trends by study design (e.g. experimental vs. cross-sectional).

\section{Measures of child snacking}

We summarize characteristics of measures used to assess child snacking in Table 4 . A wide variety of measures were used, with little consistency across the literature. The vast majority of studies used self-report to assess child snacking behaviors $(n=39)$, with caregivers frequently reporting on their child's intake $(n=20)$. Nearly half the time, a food frequency questionnaire (FFQ) was used to assess snacking ( $n=22,46.8 \%$ ), with survey tools used less frequently $(n=14)$. Open-ended tools (e.g. 24 -h recalls) were rarely used.

Most studies adapted an existing tool $(n=33,70.2 \%)$; fewer reported the use of a validated tool to assess their particular age group $(n=10,21.3 \%)$. The definition of snacking or snack intake varied greatly across measures. Since FFQs were employed often, it is not surprising that many studies defined individual food items as "snacks." However, snacks were also defined categorically based on healthy or nutritional characteristics (e.g. "junk food", "sweets", "dessert", "unhealthy", "energy dense"), or in other ways (e.g. "excessive snacking: eating between meals and at night" [63]).

Although snacks were typically measured as individual food items, they were often grouped together in a variety of ways post-hoc and then defined as snacks during analysis $(n=26,55.3 \%)$. For example, a FFQ might assess child consumption of cookies, chips, and soda as separate food items, but during subsequent analysis, the author(s) would group them together and label them as "energydense snacks." Studies of EAH $(n=6)$ were often laboratory-based and presented children with a specific set of foods, sometimes described as palatable snack foods, to evaluate children's satiety $[40,43,48,52,57,62]$. Three studies did not provide any definition of snacks and left it to the caregiver or child to determine what this word meant (e.g. "How often do you give your child snacks...").

Timing was not consistently assessed as a factor used to define a snack (i.e. chips eaten between meals vs. with lunch) during measurement or analysis. More than half the time ( $\mathrm{n}=26,55.3 \%$ ) beverages would be included in the definition of snack (e.g. soda and chips combined together as "unhealthy snacks"), but only 2 studies distinguished between beverages consumed during or between meal times. Consequently, a soda consumed with lunch could not be distinguished from a soda consumed with chips during a snack.

Frequency of snacking was the factor most often assessed ( $n=38,80.9 \%$ ), but some studies also evaluated total energy intake from snacks or child snack preferences. In rare cases, fat intake was estimated. No studies reported on snack context (e.g. where or precisely when snacking occurred) and only one described parent rationale/purpose for providing snacks.

\section{Discussion}

The aim of this systematic review was to describe how food parenting behaviors were described in the context of child snacking in quantitative studies published between 1980 and 2017. We also sought to identify how child snacking was operationalized in studies that examined food parenting and describe the demographic characteristics of study participants present in this field of research. Using evidence-based, replicable methods, we found that most studies were of good quality and reported cross-sectional findings utilizing samples that contained mostly white, college educated, female caregivers who self-reported their food parenting behaviors and their children's snack behaviors. Dietary assessment was selfreported in 3 out of 4 studies, typically using abbreviated food frequency questionnaires or brief survey items. No 


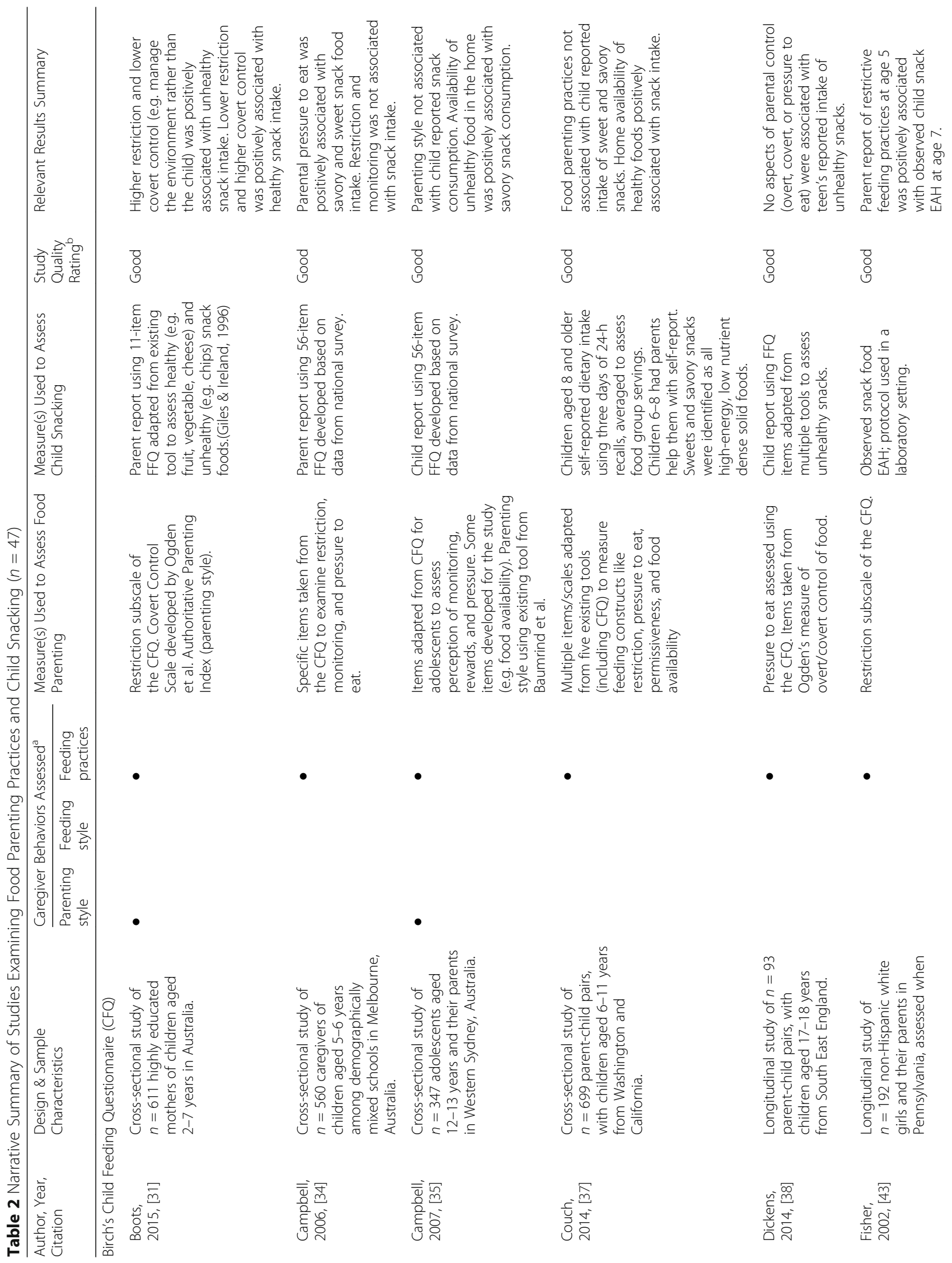




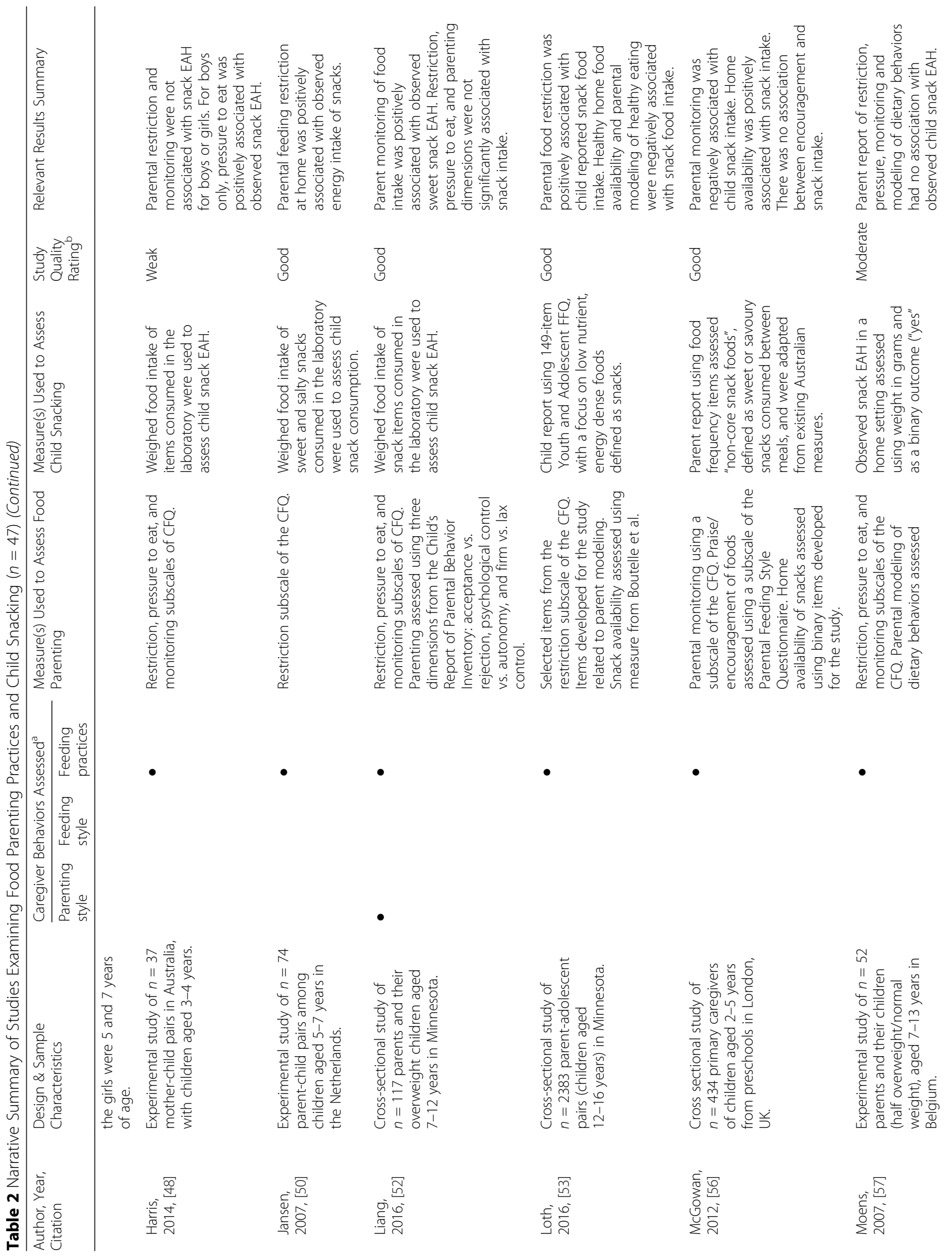




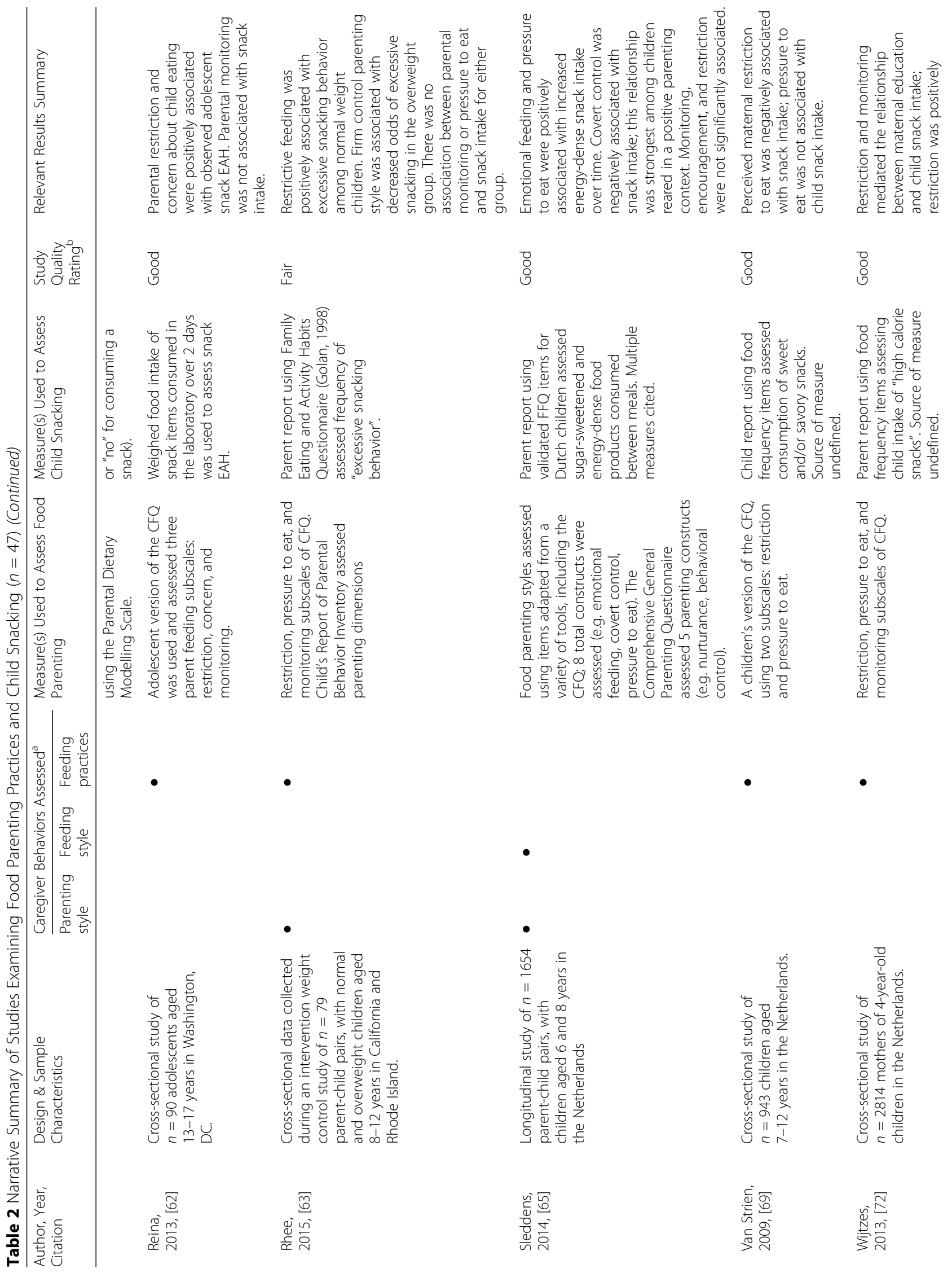




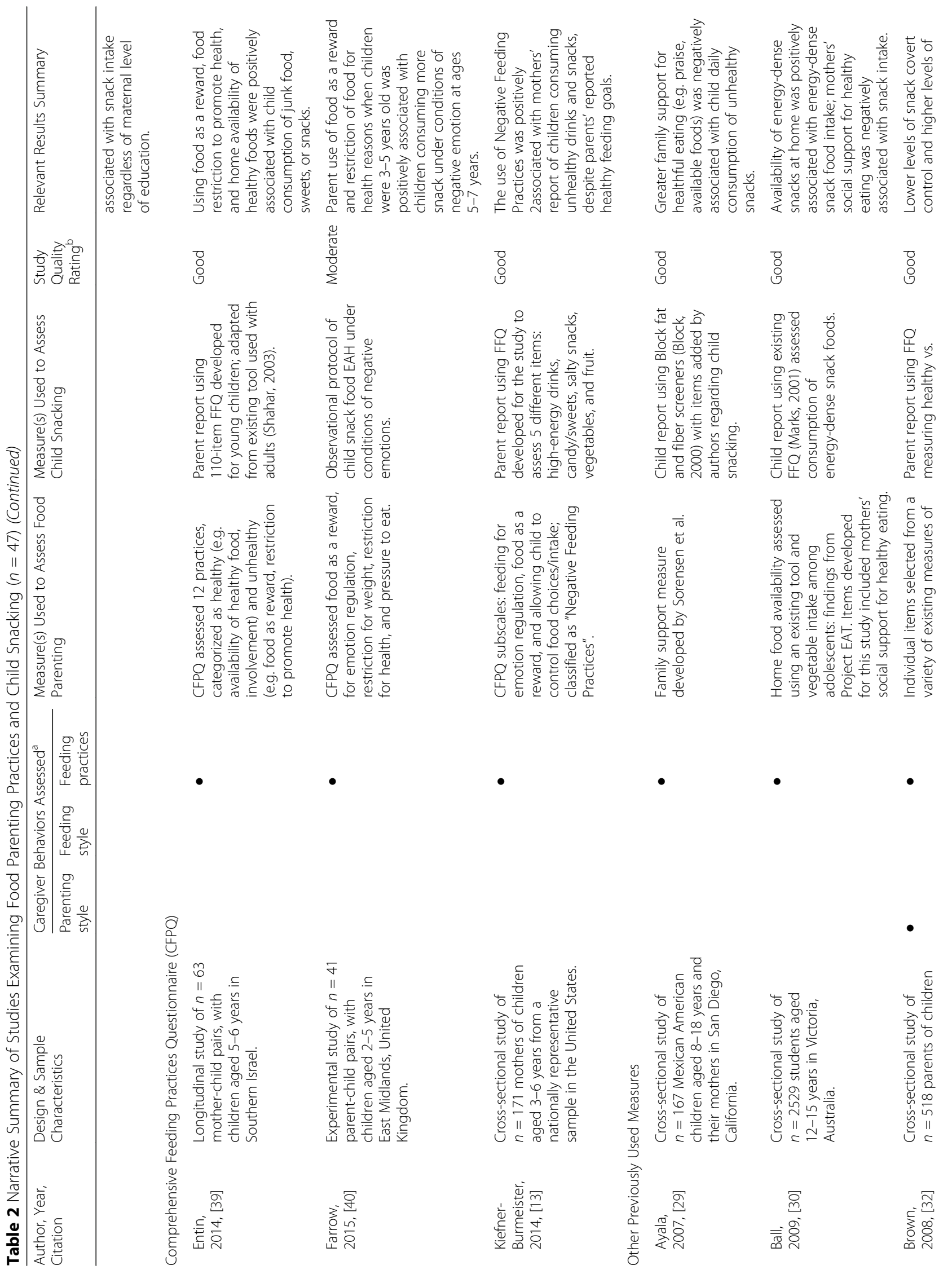




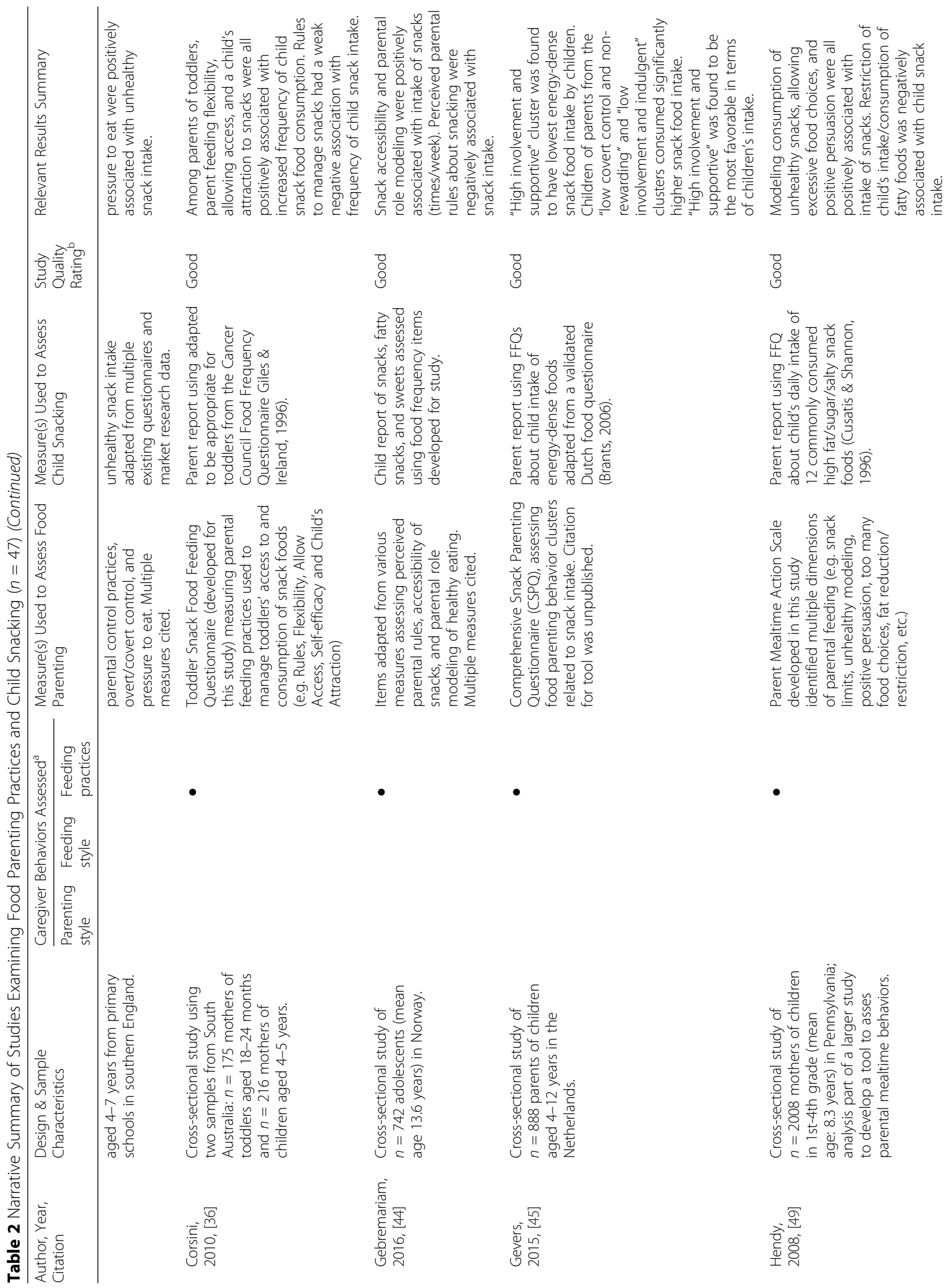




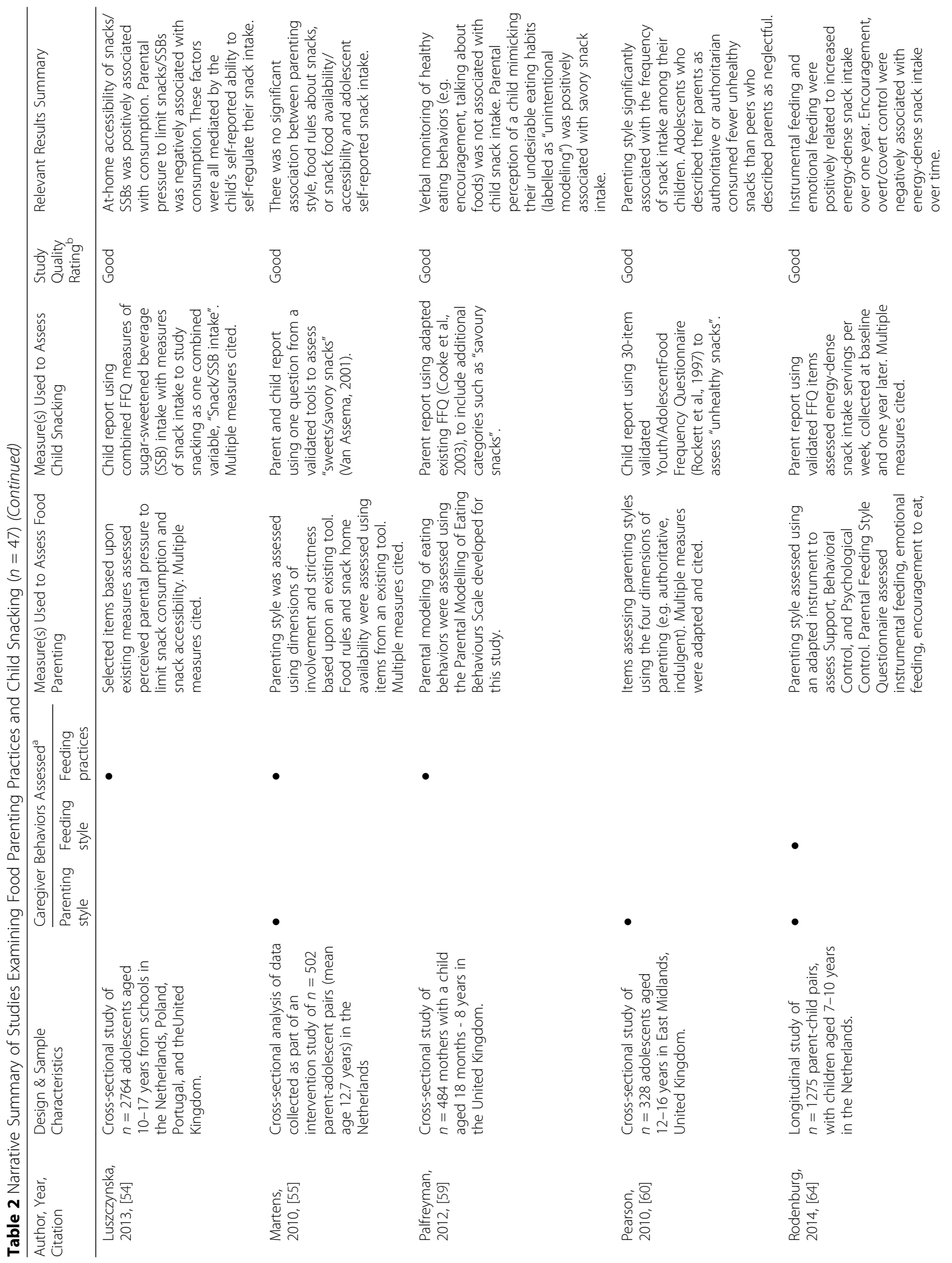




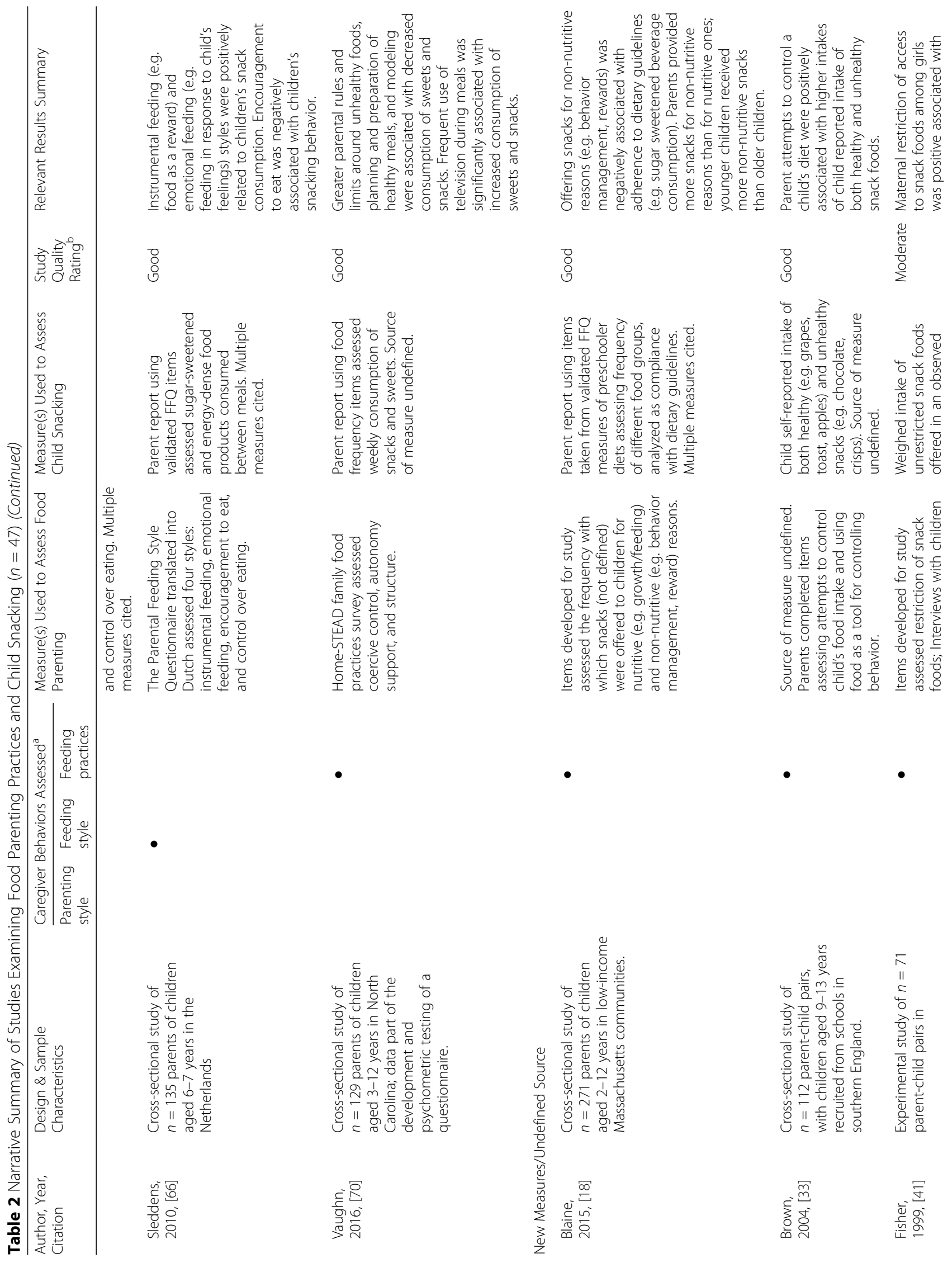




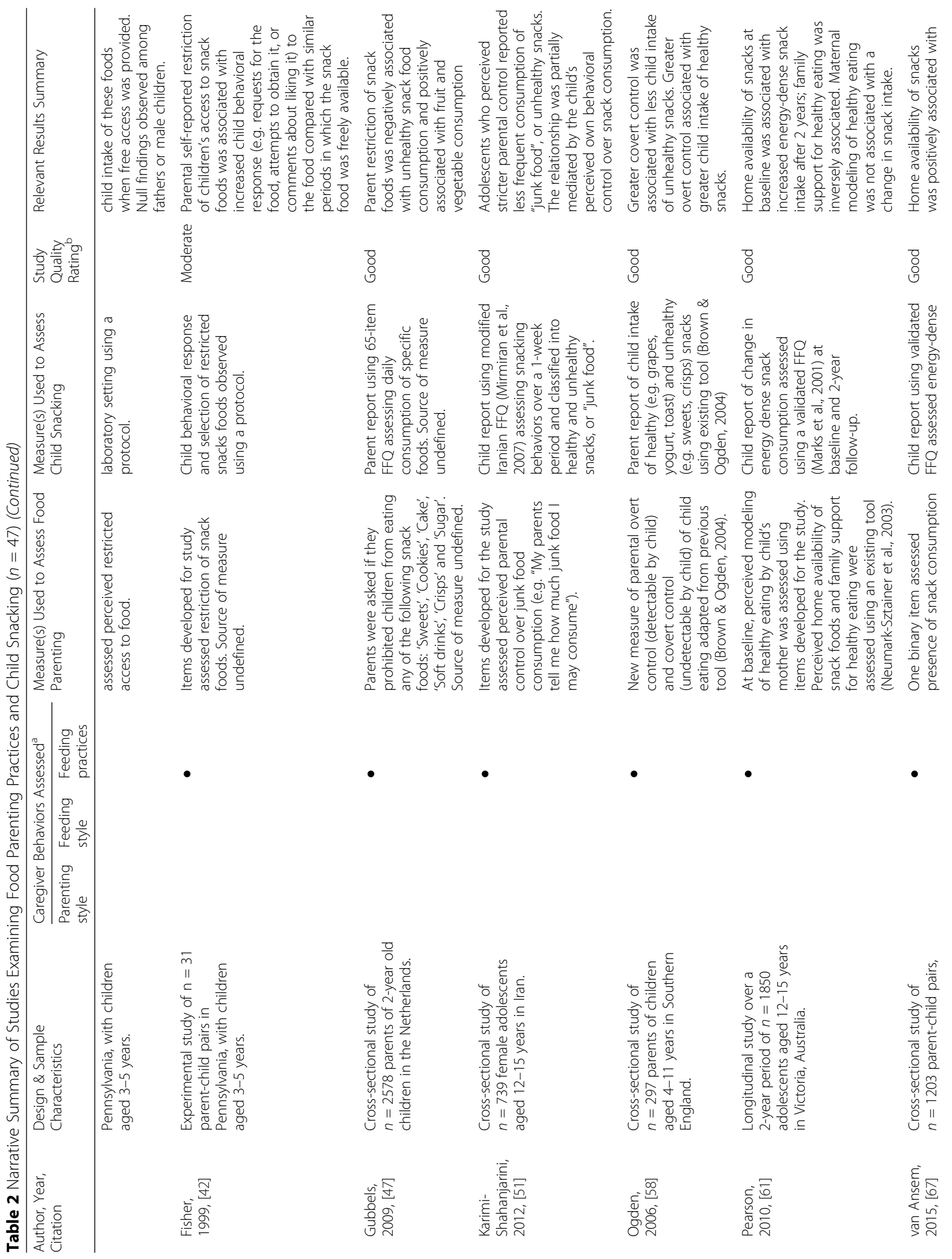




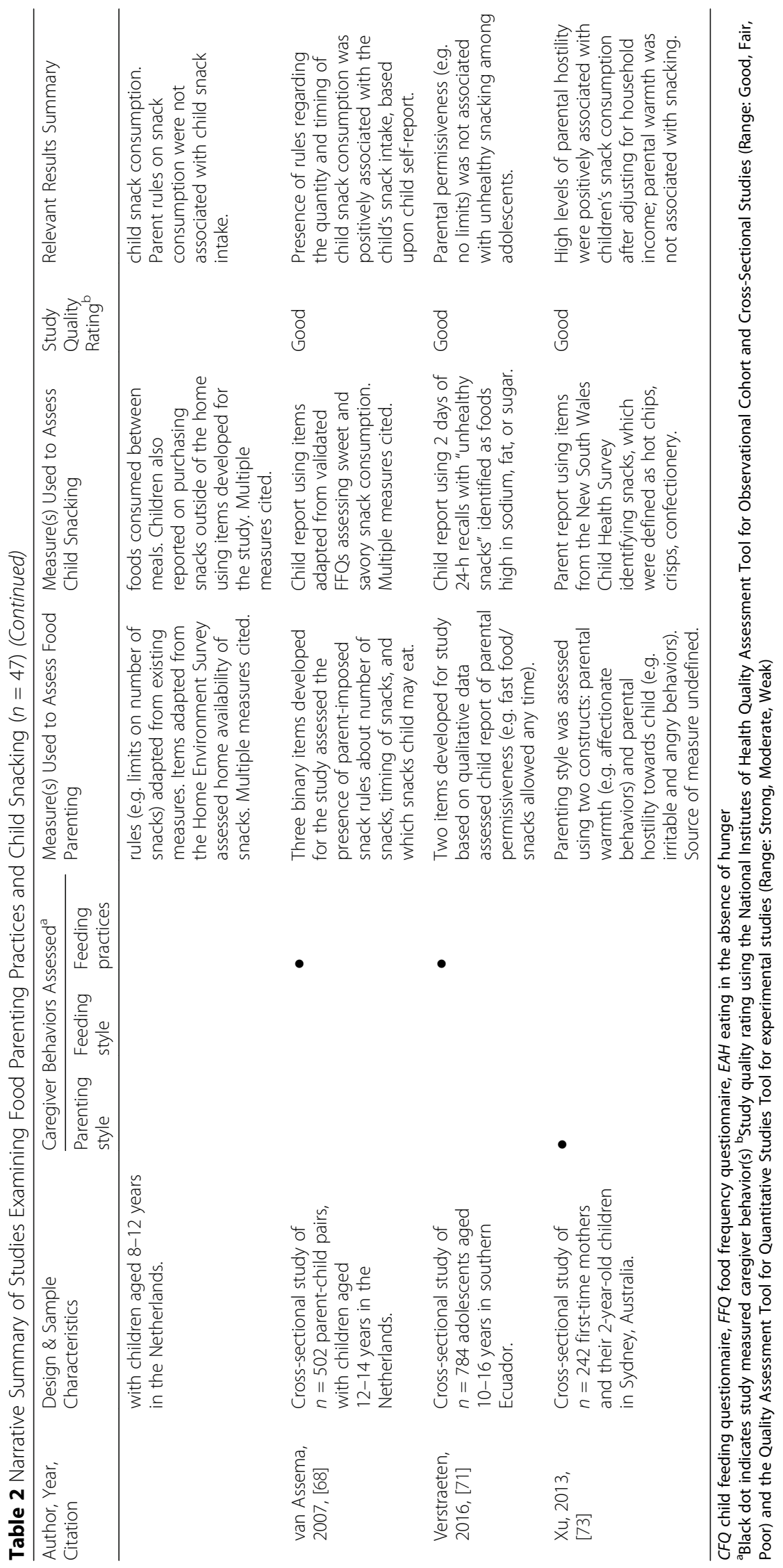


Table 3 Characteristics of $n=47$ Eligible Studies of Food Parenting and Child Snacking Published Between 1980 and 2017

\begin{tabular}{|c|c|c|}
\hline \multicolumn{3}{|l|}{ Year of Study Publication (n, \%) } \\
\hline Prior to 2000 & 2 & 4.3 \\
\hline $2000-2004$ & 2 & 4.3 \\
\hline $2005-2009$ & 12 & 25.5 \\
\hline 2010-2014 & 19 & 40.4 \\
\hline 2015-present & 12 & 25.5 \\
\hline \multicolumn{3}{|l|}{ Country (n, \%) } \\
\hline United States & 14 & 29.8 \\
\hline The Netherlands & 12 & 25.5 \\
\hline Australia & 8 & 17.0 \\
\hline United Kingdom & 8 & 17.0 \\
\hline Other & 5 & 10.6 \\
\hline \multicolumn{3}{|l|}{ Study Design (n, \%) } \\
\hline Cross-sectional & 34 & 72.3 \\
\hline Longitudinal & 6 & 12.8 \\
\hline Experimental & 7 & 14.9 \\
\hline \multicolumn{3}{|l|}{ Participants Recruited (n, \%) } \\
\hline Caregiver only & 15 & 31.9 \\
\hline Caregiver-child dyad & 21 & 44.7 \\
\hline Child only & 11 & 23.4 \\
\hline Number of Participants/Dyads (mean, SD) & 693 & 789 \\
\hline \multicolumn{3}{|l|}{ Age Ranges of Children Included in Study (n, \%) } \\
\hline Preschool ( $2-5$ years) & 20 & 42.6 \\
\hline Elementary (6-10 years) & 30 & 63.8 \\
\hline Middle School (11-13 years) & 21 & 44.7 \\
\hline High School (14-18 years) & 10 & 21.3 \\
\hline \multicolumn{3}{|l|}{ Reported Caregiver Attributes (n, \%) } \\
\hline Caregiver Race/Ethnicity & 20 & 42.6 \\
\hline Non-white participants $\geq 60 \%$ sample $^{a}$ & 6 & 30.0 \\
\hline Caregiver Gender & 29 & 55.3 \\
\hline Female-only sample & 12 & 41.3 \\
\hline Female participants $\geq 80 \%$ sample $^{\mathrm{b}}$ & 26 & 89.6 \\
\hline Fathers explicitly identified in sample ${ }^{b}$ & 10 & 34.5 \\
\hline Caregiver Level of Education & 34 & 72.3 \\
\hline College educated $\geq 60 \%$ sample ${ }^{c}$ & 23 & 67.6 \\
\hline
\end{tabular}

${ }^{a}$ Among participants that reported caregiver race/ethnicity

${ }^{\mathrm{b}}$ Among participants that distinguished between male and female caregivers

'Among participants that reported caregiver level of education

noticeable differences in trends based on feeding practices versus feeding or parenting styles were observed. There was a notable range in the measurement of types of food parenting practices and in the definition of child snacking, thus creating opportunities for improvement in future exploration of these topics. Restrictive feeding and access to unhealthy foods were most consistently associated with increases in children's snack intake, though the frequency of cross-sectional study designs limits the ability to determine causality. Few studies described autonomysupporting (e.g. praise, encouragement) or permissive (e.g. feeding to comfort) food parenting behaviors.

\section{Inconsistent definition of snacks}

Describing child snack intake presents several challenges. First, there appears to be no consensus on a universally accepted definition of child snacking in the literature we examined. Snacks were described both as a food type and as foods consumed in between meals. In most studies the word "snack" was a catch-all phrase to describe energydense, nutrient poor food types similar to "junk food"; few studies distinguished between unhealthy (e.g. chips, cookies) and healthy snacks (e.g. fruits and vegetables) [31, 58, 59]. Additionally, multiple dimensions were included in the definitions: half of the studies included beverages as snacks, while one third specified the timing when a snack food was consumed (e.g. between meals).

Another measurement challenge is that many studies defined "snacks" post-hoc, meaning the definition of snacks was often developed after data were collected, introducing possible bias depending on how or why certain foods were grouped together (e.g. relevance in the diet, statistical viability). There was great variation regarding which unhealthy foods were included or excluded across studies of similar populations. Additionally, beverages, though likely consumed alongside snack foods, often received their own separate category for analysis since timing of their intake was not routinely assessed.

Our findings that snacking definitions vary within food parenting literature are reflected elsewhere. A 2010 review of general snacking definitions concluded that studying the impact of snacking on various dietary and health outcomes was limited by the variation in definitions [76]. In another review of child snacking patterns, authors reported limited evidence of association between snacking behaviors and weight status, but emphasized that methodological limitations in the measurement of snacking might have severely limited their ability to conduct the analysis [7].

Relationship between food parenting and child snacking Despite a doubling in the number of studies describing food parenting and child snacking over the previous decade, the lack of consistency in methodology limits generalizability of findings across studies. On one hand, some of our findings appear consistent with existing literature on food parenting and general dietary intake. We found that restriction was positively associated with child snack intake in a majority of studies, which included experimental and cross-sectional designs. In other studies of food parenting, restriction of food has been linked with 


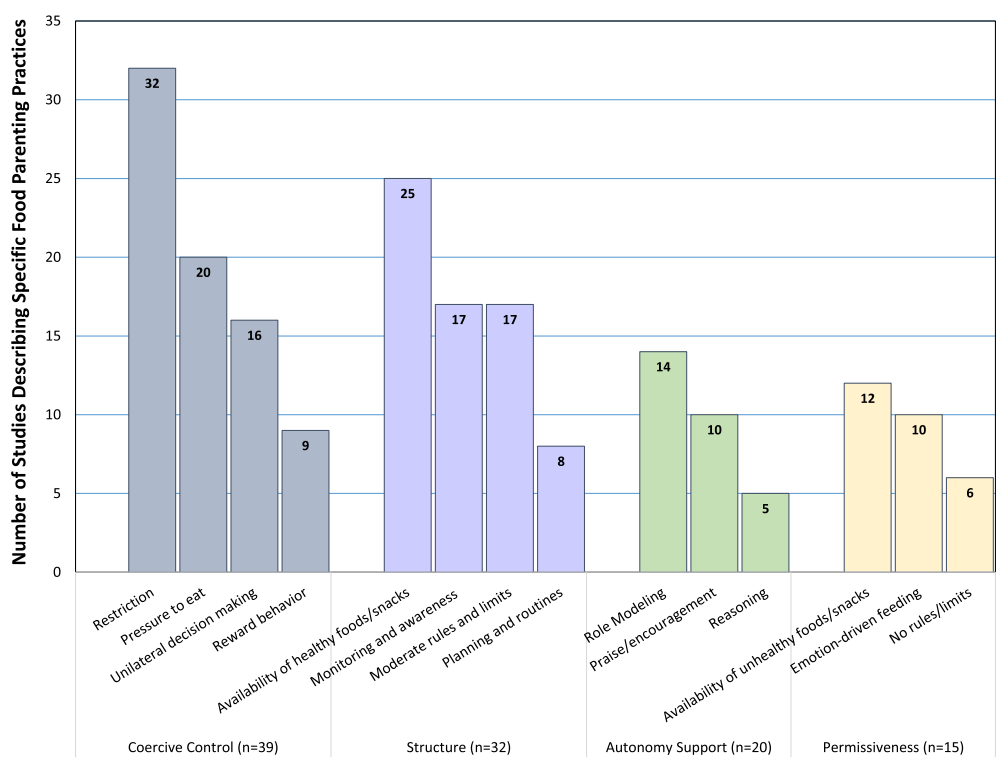

Fig. 2 Number of Studies Describing Various Food Parenting Practices in the Context of Child Snacking $(n=41)$. The total number of studies that described specific food parenting practices related to child snacking. Practices are arranged within 4 dimensions of child snack feeding derived from a theoretically guided conceptual model of food parenting around child snacking [14]

both increased caloric intake and elevated body mass index in children $[11,77]$. The underlying basis for this association is likely bidirectional, complex, and mediated by multiple factors such as a child's weight status (e.g. parents may restrict out of concern if a child is overweight). Additionally, how parents restrict (i.e. with warmth and supportive structure versus with hostility and coercive controlling practices), which may lead children to more disinhibited eating and interest in high-calorie, or "off limits" foods [10, 77]. We also found that home availability of unhealthy foods was positively associated with snack intake in 10 out of 11 studies. The home food environment has been discussed as an important risk factor for childhood obesity. However, it is not clear if this is explicitly due to the presence of the food or represents a proxy, such as role modeling or that fact that parental food and beverage intake strongly predicts that of their children $[78,79]$. Our review did not yield enough studies of parental role modeling using consistent methods $(n=2)$ to determine what impact it might have on child snacking.

Mixed findings were obtained regarding associations of pressure to eat and snacking. In the wider literature on child feeding, parental pressure to eat has been associated with both lower energy intake and body mass index in children in some studies, and increased energy intake in others, possibly because parents may be trying to encourage underweight or picky children to eat $[22,80,81]$. It is also possible that this construct is less

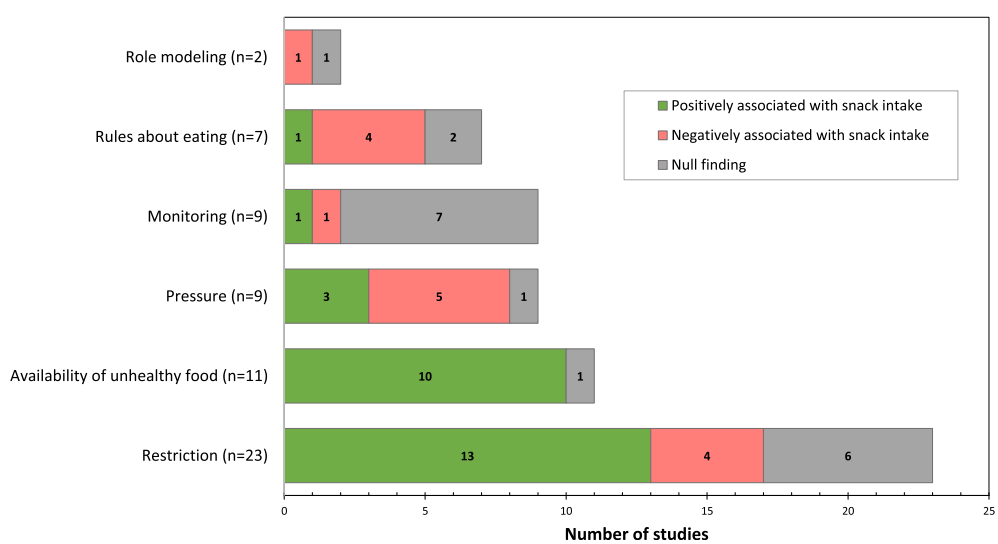

Fig. 3 Summary of Commonly Described Food Parenting Practices and Their Association with Child Snack Intake $(n=33)$. Number of studies describing positive, negative, or null associations between specific food parenting practices and child snack intake 
Table 4 Characteristics of Child Snacking Measures

\begin{tabular}{|c|c|c|}
\hline & (n) & $\%$ \\
\hline \multicolumn{3}{|l|}{ Source of Child Snacking Data } \\
\hline Parent report & 20 & 42.6 \\
\hline Child report & 17 & 36.2 \\
\hline Both parent and child reports & 2 & 4.3 \\
\hline Observed & 8 & 17.0 \\
\hline \multicolumn{3}{|l|}{ Type of Instrument } \\
\hline Food Frequency Questionnaire & 22 & 46.8 \\
\hline Survey items & 14 & 29.8 \\
\hline Observed/weighed intake & 9 & 19.1 \\
\hline 24-Hour Recall & 2 & 4.3 \\
\hline \multicolumn{3}{|l|}{ Use of Existing Measure } \\
\hline Adapted from existing measure & 33 & 70.2 \\
\hline Reported use of "validated" measure & 10 & 21.3 \\
\hline Items developed for study & 9 & 19.1 \\
\hline Source of measure undefined & 5 & 10.6 \\
\hline \multicolumn{3}{|l|}{ Measure of Snacking } \\
\hline Specific food item (e.g. chips, soda, cookies) & 34 & 72.3 \\
\hline Categorical (e.g. "desserts", "salty", "unhealthy" foods) & 8 & 17.0 \\
\hline "Snacks" - word undefined ${ }^{\mathrm{a}}$ & 3 & 6.4 \\
\hline Other & 2 & 4.3 \\
\hline \multicolumn{3}{|l|}{ Snack Intake Defined In Analysis } \\
\hline Same as in the measure & 21 & 44.6 \\
\hline Defined post-hoc (e.g. group specific foods as "snack") & 26 & 55.3 \\
\hline \multicolumn{3}{|l|}{ Specificity in Definition of "Snack" } \\
\hline Beverages included (e.g. soda is a snack food) & 26 & 55.3 \\
\hline Timing (e.g. foods consumed between meals) & 14 & 29.7 \\
\hline Healthy snacks identified (e.g. a fruit could be a snack) & 3 & 6.4 \\
\hline $\begin{array}{l}\text { Beverage timing (e.g. differentiate soda with snack vs. } \\
\text { dinner) }\end{array}$ & 2 & 4.3 \\
\hline \multicolumn{3}{|l|}{ Snacking Factors Assessed } \\
\hline Frequency & 38 & 80.9 \\
\hline Energy intake (total calories) & 11 & 23.4 \\
\hline Child preference & 2 & 4.3 \\
\hline Rationale (e.g. why snack offered) & 1 & 2.1 \\
\hline Fat intake & 1 & 2.1 \\
\hline
\end{tabular}

a Used the word "snack" in the instrument (e.g. "When do you give snacks"..) without a definition

utilized in the context of child snacking, as parents may be more likely to pressure children to eat foods deemed "healthy." This is consistent with a qualitative conceptual study of food parenting around child snacking that found very few low-income parents identified pressure as part of their schemas around snacking $[10,14]$.

We also found monitoring food intake bore null findings in a majority of studies [81]. One possible reason for this may be that monitoring can be characterized as controlling when paired with other behaviors (e.g. restriction) and may be positive if it is paired with structure-supporting behaviors (e.g. reasonable limits, offering healthy foods) [14]. Additionally, few studies employed measures that focused specifically on snack food parenting, which may reduce their relevance for some food parenting practices.

Although a number of validated tools exist to assess food parenting practices [9], few studies in our review utilized complete measures, and instead took specific items or partial subscales from tools like the Child Feeding Questionnaire [74] to assess specific controlling feeding practices (e.g. restriction). Measurement of food parenting presents a challenge, as many child feeding tools have numerous items and subscales, which affects participant burden. However, adaptation presents a threat to validity, as psychometric properties of validated scales do not necessarily apply when subsets of items are administered. It is possible such adaptations contributed to mixed findings when we examined associations between food parenting practices and child snacking.

\section{Recommendations for future research \\ Recommendation \#1: Investigate parenting specific to child snacking}

In general, the literature presents negative food parenting practices like restriction and pressure to eat, compared with role modeling, healthy limit-setting, or encouragement. Therefore, it would be beneficial for future studies to include positive parenting behaviors to identify how these can be supported and translated into public health interventions. At present, there are a limited number of tools that exist to measure food parenting specific to snacking. The Toddler Snack Food Feeding Questionnaire [36] assesses both negative and positive food parenting dimensions and is validated for use with caregivers of children aged 1-2 years. The Parent Mealtime Action Scale [49] measures overall parent mealtime behavior, but does present two dimensions that are specifically positive and snack focused (e.g. snack limits and snack modeling); this tool was validated with caregivers of children in 1st-4th grade (aged 6-9 years). In the future, it would be beneficial to expand these measures or create a new tool to assess the full spectrum of food parenting practices around snacking.

\section{Recommendation \#2: Increase diversity in caregiver perspectives}

Our review found that mothers almost exclusively represented caregivers of interest with respect to food parenting around child snacking. We noted that a vast majority of studies either did not mention fathers or male caregivers (e.g. stepfather, live-in partner of mother), and if mentioned, they comprised $10 \%$ or less of samples. Increasingly, men are playing a greater role in child rearing, and 
their absence in studies of food parenting [28] and childhood obesity-related risk factors [82, 83] presents a major gap in the literature. Thus, it is important to intentionally recruit men in studies of snack food parenting and examine whether their practices conflict with or support that of female partners, or female caregivers as a whole. Future studies should define a parent or caregiver, and clearly convey the number of female and male caregivers included in the sample. Additionally, there is evidence that other informal caregivers, such as grandparents, may play an increasingly important role in the provision of snacks to children [84, 85].

Caregivers in the studies reviewed were typically white and highly educated, consistent with other literature exploring parenting and obesity-related risk factors in children $[11,86]$. In light of the health disparities that lowincome children from racial/ethnic minority groups face with respect to food quality, healthy food availability, and childhood obesity [87, 88], an intentional approach towards recruiting diverse families is warranted. Additionally, recent qualitative work suggests that low-income parents may use snack foods specifically as an affordable way to comfort children or provide treats in the absence of other costly pleasures (e.g. vacations, movies) $[17,89,90]$. Therefore, more quantitative studies are also needed to identify differences in food parenting intentions and practices based upon such sociodemographic factors.

\section{Recommendation \#3: Describe child snacking contexts and purposes}

The context in which child snacking occurs is poorly defined in the literature. Although most quantitative studies described the number of snacks children consume, only one described the purpose, or parent rationale for providing snacks (e.g. reward, to promote health) [18]. No studies in our review described the physical context or timing in which snacking occurred. There is reason to believe that timing may also be an important factor, as a recent review of American children's snacking patterns found that afternoon snacks might be more energy dense and nutrition-poor that morning snacks [91].

One qualitative study of low-income multi-ethnic caregivers of 2-5-year-old children provides additional insight, revealed that snacking timing and location were important parts of their definition of a snack [92]. Parents reported that children were often fed in response to environmental stimuli (e.g. ice cream truck, while grocery shopping) or that physical context dictated their child's snacking habits (e.g. whenever the TV was turned on) $[14,89]$. Another analysis from the same study found that nutritional quality of snacks varied greatly based upon self-reported purposes; children received healthier snacks when parents were addressing their hunger and less healthy snacks when they were being rewarded [93]. Therefore, understanding both context and the underlying purpose of snack feeding is critical to developing effective public health messages for parents and may also help to identify environmental triggers for food parenting practices that are most obesogenic.

\section{Recommendation \#4: Move toward more consistent terminology and detailed definitions around child snacking}

The current heterogeneity in definitions of child snacking limits the field in progressing towards greater understanding of snacking behaviors. Given that measurement of snacking varies based upon populations, research aims, and methodologies, it is not likely feasible to provide one universal definition of child snack foods. However, we propose the use of consistent terminology and dimensions of snacking (Table 5).

Primarily, we suggest that snack foods be defined as foods or beverages consumed between meals in order to standardize language across studies. Within this definition, nutrient-rich items like fruits, vegetables, and whole grains consumed between meals may also qualify as snacks, thus leading the field towards including more healthful eating behaviors in research. If items are defined as "unhealthy" snack foods, we recommend providing explicit details about all food/beverages assessed and the specific rationale for such categorization. Nutrientpoor foods assessed without the context of the timing (e.g. junk food or soda consumed at any time of day) would not be considered snack foods within this proposed definition.

Some studies may use qualitative research to define snacking within a population in order to identify the full range of foods consumed between meals as "snacks". For example, one caregiver-defined definition of snacking

Table 5 Suggested Standardized Terminology and Definitions for Future Research on Child Snacking

\begin{tabular}{ll}
\hline Terminology & Suggested Definition \\
\hline Snack foods (and beverages, if applicable) & $\begin{array}{l}\text { Foods and/or beverages that are consumed by children between meals. Researchers may } \\
\text { provide their own specific qualifiers (e.g. "energy-dense snack foods", "sugary snack foods") } \\
\text { along with explicit criteria for these classifications. Terminology may be shortened to "snack" } \\
\text { or "snacks" after it has been defined. }\end{array}$ \\
$\begin{array}{ll}\text { Snacking occasions } & \text { The number of between-meal eating episodes in a given day. } \\
\text { Snacking purposes } & \text { Reasons that parents offer foods between meals (e.g. child request, reward, special occasion, routine). } \\
\text { Snacking contexts } & \text { Places where between-meal eating occurs (e.g. at home, in the car, at church). }\end{array}$
\end{tabular}


among preschool-aged children that was recently presented by Younginer et al. [92] is, "A small portion of food that is given in-between meals, frequently with an intention of reducing or preventing hunger until the next mealtime." When parents in this population were asked about why or when they give their children "snacks", this definition is useful to properly interpret the findings.

Measuring all dimensions of snacking certainly has implications for participant burden and is not likely to be feasible in most studies. A smaller-scale study that utilizes high-burden measures to validate a lower burden questionnaire-based assessment of various snacking dimensions would be a promising strategy to enable largescale assessment of associations with food parenting and other factors in the future.

\section{Strengths and limitations}

Our review presents several strengths. First, we provide transparent and replicable methods using PRISMA guidelines. We provide our search protocol, detailed search strategy, and data extraction tool with our findings. We also utilized double coding of all data extracted, including screening and full-text analysis in order to increase validity of our results. Additionally, we built our review upon a theoretically guided conceptual model of food parenting around child snacking so that our findings could be presented in the context of the current momentum within the literature. We use the same terminology and definitions of food parenting practices presented in the model in order to maximize construct operationalization.

Our review also has limitations. Due to the vast number of studies requiring screening, we did not review the bibliographies of full-texts to identify additional articles. We also did not include grey literature in our search, which could have increased the number of possible publications. The cross-sectional design of most studies we present also limits our ability to assess causality or temporality of the relationship between food parenting and child snacking. Due to the lack of standardization across measures of food parenting and child snacking, our review is limited to a descriptive, narrative summary of the state of the research, rather than a meta-analysis. However, our hope is that providing recommendations to improve future methodology will allow for such analysis in the future.

\section{Conclusions}

Snacking among children is nearly universal and significantly contributes to children's intake of energy and other nutrients. Parents play an important role in shaping children's dietary behaviors, including snacking. This study is the first to systematically describe food parenting specifically in the context of child snacking.
Restrictive feeding and child access to unhealthy foods have been most consistently associated with increases in children's snack intake. Pressure to eat and monitoring have yielded mixed and null findings. With mounting attention paid to the role of child snacking on obesity risk in recent years, a universal definition of snacking that addresses both food type and timing is needed to maximize generalizability across studies and advance findings within the field. Future research should include positive food parenting behaviors around child snacking that may be used as targets for health promotion.

\section{Additional files}

Additional file 1: Preferred Reporting Items for Systematic Reviews and Meta-Analyses (PRISMA) checklist indicating standardized procedures for data collection and analysis (DOCX $27 \mathrm{~kb}$ )

Additional file 2: Protocol containing inclusion and exclusion criteria, along with an electronic search strategy for the study (DOCX 16 kb)

Additional file 3: Pre-defined list of items to be coded from articles that were included in the review. Includes the complete tool used in SurveyGizmo (DOCX 24 kb)

\section{Abbreviations}

CFQ: Child Feeding Questionnaire; EAH: Eating in the absence of hunger: FFQs: Food Frequency Questionnaires; PRISMA: Preferred Reporting Items for Systematic Reviews and Meta-Analyses

\section{Acknowledgements \\ None.}

Funding

No funding sources to declare.

\section{Availability of data and materials}

The data files used during the current study are available from the corresponding author upon reasonable request.

Authors' contributions

REB designed the study, contributed to article screening, data extraction, coding, and analysis, and drafted the complete manuscript. AK screened abstracts, extracted and coded data, and assisted in analysis. KKD contributed to study conceptualization and design. RK contributed to article identification, data extraction, and coding. JOF contributed to study conceptualization, design, development of the coding scheme, and interpretation of the data. All authors read, provided edits, and approved the final manuscript.

Ethics approval and consent to participate

Not applicable.

Consent for publication

Not applicable.

Competing interests

The authors declare that they have no competing interests.

\section{Publisher's Note}

Springer Nature remains neutral with regard to jurisdictional claims in published maps and institutional affiliations.

\section{Author details}

'Department of Family and Consumer Sciences, California State University, Long Beach, 1250 Bellflower Blvd, FCS FA-15, Long Beach, CA 90840-0501, USA. ${ }^{2}$ Department of Social and Behavioral Sciences, Center for Obesity Research and Education, Temple University, 3223 N. Broad Street, Suite 175, 
Philadelphia, PA 19140, USA. ${ }^{3}$ Department of Nutrition, Harvard T.H. Chan School of Public Health, 655 Huntington Ave, Boston, MA 02115, USA. ${ }^{4}$ Department of Global Health \& Population, Harvard T.H. Chan School of Public Health, 655 Huntington Ave, Boston, MA 02115, USA.

\section{Received: 1 June 2017 Accepted: 3 October 2017}

Published online: 03 November 2017

\section{References}

1. Ogden CL, Carroll MD, Lawman HG, Fryar CD, Kruszon-Moran D, Kit BK, Flegal KM. Trends in obesity prevalence among children and adolescents in the United States, 1988-1994 through 2013-2014. JAMA. 2016;315:2292-9.

2. Wang $Y$, Lim H. The global childhood obesity epidemic and the association between socio-economic status and childhood obesity. Int Rev Psychiatry. 2012:24:176-88.

3. Ford CN, Slining MM, Popkin BM. Trends in dietary intake among US 2-to 6year-old children, 1989-2008. J Am Diet. 2013;113:35-42. e36

4. Adair LS, Popkin BM. Are child eating patterns being transformed globally? Obesity. 2005;13:1281-99.

5. Piernas C, Popkin BM. Trends in snacking among US children. Health Aff. 2010;29:398-404.

6. Samuelson G. Dietary habits and nutritional status in adolescents over Europe. An overview of current studies in the Nordic countries. Eur J Clin Nutr. 2000;54:S21.

7. Larson N, Story M. A review of snacking patterns among children and adolescents: what are the implications of snacking for weight status? Child Obes. 2013;9:104-15.

8. Evans EW, Jacques PF, Dallal GE, Sacheck J, Must A. The role of eating frequency on total energy intake and diet quality in a low-income, racially diverse sample of schoolchildren. Public Health Nutr. 2015;18:474-81.

9. Vaughn AE, Tabak RG, Bryant MJ, Ward DS. Measuring parent food practices: a systematic review of existing measures and examination of instruments. Int J Behav Nutr Phys Act. 2013;10:61.

10. Vollmer RL, Mobley AR. Parenting styles, feeding styles, and their influence on child obesogenic behaviors and body weight. A review. Appetite. 2013;71:232-41

11. Sleddens EF, Gerards SM, Thijs C, NK VRIES, Kremers SP. General parenting, childhood overweight and obesity-inducing behaviors: a review. Int J Pediatr Obes. 2011;6:e12-27.

12. Dovey TM, Staples PA, Gibson EL, Halford JC. Food neophobia and 'picky/ fussy'eating in children: a review. Appetite. 2008:50:181-93.

13. Kiefner-Burmeister AE, Hoffmann DA, Meers MR, Koball AM, MusherEizenman DR. Food consumption by young children: a function of parental feeding goals and practices. Appetite. 2014;74:6-11.

14. Davison KK, Blake CE, Blaine RE, Younginer NA, Orloski A, Hamtil HA, Ganter C, Bruton YP, Vaughn AE, Fisher JO. Parenting around child snacking: development of a theoretically-guided, empirically informed conceptual model. Int J Behav Nutr Phys Act. 2015;12:109.

15. Blissett J. Relationships between parenting style, feeding style and feeding practices and fruit and vegetable consumption in early childhood. Appetite. 2011;57:826-31.

16. Shloim N, Edelson LR, Martin N, Hetherington MM. Parenting styles, feeding styles, feeding practices, and weight status in 4-12 year-old children: a systematic review of the literature. Front Psychol. 2015;6:-1849.

17. Fisher JO, Wright G, Herman AN, Malhotra K, Serrano EL, Foster GD, Whitaker RC. "snacks are not food". Low-income, urban mothers' perceptions of feeding snacks to their preschool-aged children. Appetite. 2015:84:61-7.

18. Blaine RE, Fisher JO, Taveras EM, Geller AC, Rimm EB, Land T, Perkins M, Davison KK. Reasons low-income parents offer snacks to children: how feeding rationale influences snack frequency and adherence to dietary recommendations. Nutrients. 2015;7:5982-99.

19. Hughes SO, Power TG, Orlet Fisher J, Mueller S, Nicklas TA. Revisiting a neglected construct: parenting styles in a child-feeding context. Appetite. 2005:44:83-92.

20. Hughes CC, Sherman SN, Whitaker RC. How low-income mothers with overweight preschool children make sense of obesity. Qual Health Res. 2010;20:465-78.

21. Vaughn AE, Ward DS, Fisher JO, Faith MS, Hughes SO, Kremers SP, MusherEizenman DR, O'Connor TM, Patrick H, Power TG. Fundamental constructs in food parenting practices: a content map to guide future research. Nutr Rev. 2016:74:98-117.

22. Loth KA. Associations between food restriction and pressure-to-eat parenting practices and dietary intake in children: a selective review of the recent literature. Curr Nutr Rep. 2016;5:61-7.

23. Moher D, Liberati A, Tetzlaff J, Altman DG, Group P. Preferred reporting items for systematic reviews and meta-analyses: the PRISMA statement. PLoS Med. 2009;6:e1000097.

24. Cutting TM, Fisher JO, Grimm-Thomas K, Birch LL. Like mother, like daughter: familial patterns of overweight are mediated by mothers' dietary disinhibition. Am J Clin Nutr. 1999;69:608-13.

25. Grove RW. An analysis of the constant comparative method. Int J Qual Stud Educ. 1988:1:273-9.

26. National Heart L, Institute B. Quality assessment tool for observational cohort and cross-sectional studies. Bethesda: MD: National Institutes of Health, Department of Health and Human Services; 2014

27. Thomas H: Quality assessment tool for quantitative studies. Effective public health practice project Toronto: McMaster University 2003.

28. Khandpur N, Blaine RE, Fisher JO, Davison KK. Fathers' child feeding practices: a review of the evidence. Appetite. 2014;78:110-21.

29. Ayala GX, Baquero B, Arredondo EM, Campbell N, Larios S, Elder JP. Association between family variables and Mexican American children's dietary behaviors. J Nutr Educ Behav. 2007:39:62-9.

30. Ball K, MacFarlane A, Crawford D, Savige G, Andrianopoulos N, Worsley A. Can social cognitive theory constructs explain socio-economic variations in adolescent eating behaviours? A mediation analysis. Health Educ Res. 2009;24:496-506.

31. Boots SB, Tiggemann M, Corsini N, Mattiske J. Managing young children's snack food intake. The role of parenting style and feeding strategies. Appetite. 2015;92:94-101.

32. Brown KA, Ogden J, Vogele C, Gibson EL. The role of parental control practices in explaining children's diet and BMI. Appetite. 2008:50:252-9.

33. Brown R, Ogden J. Children's eating attitudes and behaviour: a study of the modelling and control theories of parental influence. Health Educ Res. 2004;19:261-71

34. Campbell KJ, Crawford DA, Ball K. Family food environment and dietary behaviors likely to promote fatness in 5-6 year-old children. Int J Obes. 2006;30:1272-80.

35. Campbell KJ, Crawford DA, Salmon J, Carver A, Garnett SP, Baur LA. Associations between the home food environment and obesity-promoting eating behaviors in adolescence. Obesity (Silver Spring). 2007;15:719-30.

36. Corsini N, Wilson C, Kettler L, Danthiir V. Development and preliminary validation of the toddler snack food feeding questionnaire. Appetite. 2010:54:570-8.

37. Couch SC, Glanz K, Zhou C, Sallis JF, Saelens BE. Home food environment in relation to Children's diet quality and weight status. J Acad Nutr Diet. 2014;

38. Dickens E, Ogden J. The role of parental control and modelling in predicting a child's diet and relationship with food after they leave home. A prospective study. Appetite. 2014;76:23-9.

39. Entin A, Kaufman-Shriqui V, Naggan L, Vardi H, Shahar DR: Parental Feeding Practices in Relation to Low Diet Quality and Obesity among LSES Children. J Am Coll Nutr 20141-9.

40. Farrow CV, Haycraft E, Blissett JM. Teaching our children when to eat: how parental feeding practices inform the development of emotional eating-a longitudinal experimental design. Am J Clin Nutr. 2015;101:908-13.

41. Fisher JO, Birch LL. Restricting access to foods and children's eating Appetite. 1999;32:405-19.

42. Fisher JO, Birch LL. Restricting access to palatable foods affects children's behavioral response, food selection, and intake. Am J Clin Nutr. 1999;69:1264-72

43. Fisher JO, Birch LL. Eating in the absence of hunger and overweight in girls from 5 to 7 y of age. Am J Clin Nutr. 2002;76:226-31.

44. Gebremariam MK, Henjum S, Terragni L, Torheim LE. Correlates of fruit, vegetable, soft drink, and snack intake among adolescents: the ESSENS study. Food Nutr Res. 2016:60:32512.

45. Gevers DW, Kremers SP, de Vries NK, van Assema P. Patterns of food parenting practices and Children's intake of energy-dense snack foods. Nutrients. 2015;7:4093-106

46. Gevers DW, van Assema P, Sleddens EF, de Vries NK, Kremers SP. Associations between general parenting, restrictive snacking rules, and adolescent's snack intake. The roles of fathers and mothers and interparental congruence. Appetite. 2015;87:184-91. 
47. Gubbels JS, Kremers SP, Stafleu A, Dagnelie PC, Goldbohm RA, de Vries NK, Thijs C. Diet-related restrictive parenting practices. Impact on dietary intake of 2-year-old children and interactions with child characteristics. Appetite. 2009:52:423-9.

48. Harris H, Mallan KM, Nambiar S, Daniels LA. The relationship between controlling feeding practices and boys' and girls' eating in the absence of hunger. Eat Behav. 2014;15:519-22.

49. Hendy HM, Williams KE, Camise TS, Eckman N, Hedemann A. The parent mealtime action scale (PMAS). Development and association with children's diet and weight. Appetite. 2008;52:328-39.

50. Jansen E, Mulkens S, Jansen A. Do not eat the red food!: prohibition of snacks leads to their relatively higher consumption in children. Appetite. 2007:49:572-7.

51. Karimi-Shahanjarini A, Rashidian A, Majdzadeh R, Omidvar N, Tabatabai MG, Shojaeezadeh D. Parental control and junk-food consumption: a mediating and moderating effect analysis. J Appl Soc Psychol. 2012:42:1241-65.

52. Liang J, Matheson BE, Rhee KE, Peterson CB, Rydell S, Boutelle KN. Parental control and overconsumption of snack foods in overweight and obese children. Appetite. 2016;100:181-8.

53. Loth KA, MacLehose RF, Larson N, Berge JM, Neumark-Sztainer D. Food availability, modeling and restriction: how are these different aspects of the family eating environment related to adolescent dietary intake? Appetite. 2016;96:80-6.

54. Luszczynska A, de Wit JB, de Vet E, Januszewicz A, Liszewska N, Johnson F, Pratt M, Gaspar T, de Matos MG, Stok FM. At-home environment, out-ofhome environment, snacks and sweetened beverages intake in preadolescence, early and mid-adolescence: the interplay between environment and self-regulation. J Youth Adolesc. 2013;42:1873-83.

55. Martens M, van Assema P, Knibbe R, Engels RC, Brug J. Family environmental factors do not explain differences in the behavioral effect of a healthy diet promotion program in lower vocational schools among 12to 14-year-old adolescents. Am J Health Promot. 2010;24:182-5.

56. McGowan L, Croker H, Wardle J, Cooke LJ. Environmental and individual determinants of core and non-core food and drink intake in preschool-aged children in the United Kingdom. Eur J Clin Nutr. 2012;66:322-8.

57. Moens E, Braet C. Predictors of disinhibited eating in children with and without overweight. Behav Res Ther. 2007:45:1357-68.

58. Ogden J, Reynolds R, Smith A. Expanding the concept of parental control: a role for overt and covert control in children's snacking behaviour? Appetite. 2006:47:100-6

59. Palfreyman Z, Haycraft E, Meyer C. Development of the parental Modelling of eating Behaviours scale (PARM): links with food intake among children and their mothers. Matern Child Nutr. 2012;10:617-29.

60. Pearson N, Atkin AJ, Biddle SJ, Gorely T, Edwardson C. Parenting styles, family structure and adolescent dietary behaviour. Public Health Nutr. 2010;13:1245-53.

61. Pearson N, Ball K, Crawford D. Predictors of changes in adolescents' consumption of fruits, vegetables and energy-dense snacks. $\mathrm{Br} J$ Nutr. 2010;105:795-803.

62. Reina SA, Shomaker LB, Mooreville M, Courville AB, Brady SM, Olsen C, Yanovski SZ, Tanofsky-Kraff M, Yanovski JA. Sociocultural pressures and adolescent eating in the absence of hunger. Body Image. 2013;10:182-90.

63. Rhee KE, Boutelle KN, Jelalian E, Barnes R, Dickstein S, Wing RR. Firm maternal parenting associated with decreased risk of excessive snacking in overweight children. Eat Weight Disord. 2015;20:195-203.

64. Rodenburg G, Kremers SP, Oenema A, van de Mheen D. Associations of parental feeding styles with child snacking behaviour and weight in the context of general parenting. Public Health Nutr. 2014;17:960-9.

65. Sleddens EF, Kremers SP, Stafleu A, Dagnelie PC, De Vries NK, Thijs C. Food parenting practices and child dietary behavior. Prospective relations and the moderating role of general parenting. Appetite. 2014;79:42-50.

66. Sleddens EFC, Kremers SPJ, De Vries NK, Thijs C. Relationship between parental feeding styles and eating behaviours of Dutch children aged 6-7. Appetite. 2010;54:30-6

67. van Ansem WJ, Schrijvers CT, Rodenburg G, van de Mheen D. Children's snack consumption: role of parents, peers and child snack-purchasing behaviour. Results from the INPACT study. Eur J Pub Health. 2015;25:1006-11.

68. van Assema P, Glanz K, Martens M, Brug J. Differences between parents' and adolescents' perceptions of family food rules and availability. J Nutr Educ Behav. 2007;39:84-9.

69. Van Strien T, van Niekerk R, Ouwens MA. Perceived parental food controlling practices are related to obesogenic or leptogenic child life style behaviors. Appetite. 2009;53:151-4.
70. Vaughn AE, Dearth-Wesley T, Tabak RG, Bryant M, Ward DS. Development of a Comprehensive Assessment of Food Parenting Practices: The Home SelfAdministered Tool for Environmental Assessment of Activity and Diet Family Food Practices Survey. Journal of the Academy of Nutrition and Dietetics. 2017;117(2):214-27.

71. Verstraeten R, Leroy JL, Pieniak Z, Ochoa-Aviles A, Holdsworth M, Verbeke W, Maes L, Kolsteren P. Individual and environmental factors influencing Adolescents' dietary behavior in low- and middle-income settings. PLoS One. 2016;11:e0157744.

72. Wijtzes Al, Jansen W, Jansen PW, Jaddoe WW, Hofman A, Raat H. Maternal educational level and preschool children's consumption of high-calorie snacks and sugar-containing beverages: mediation by the family food environment. Int J Prev Med. 2013;57:607-12.

73. Xu H, Wen LM, Rissel C, Flood VM, Baur LA. Parenting style and dietary behaviour of young children. Findings from the healthy beginnings trial. Appetite. 2013;71:171-7.

74. Birch LL, Fisher J, Grimm-Thomas K, Markey C, Sawyer R, Johnson SL. Confirmatory factor analysis of the child feeding questionnaire: a measure of parental attitudes, beliefs and practices about child feeding and obesity proneness. Appetite. 2001;36:201-10.

75. Musher-Eizenman D, Holub S. Comprehensive feeding practices questionnaire: validation of a new measure of parental feeding practices. J Pediatr Psychol. 2007;32:960-72.

76. Johnson GH, Anderson GH. Snacking definitions: impact on interpretation of the literature and dietary recommendations. Crit Rev Food Sci Nutr. 2010;50:848-71

77. Joyce JL, Zimmer-Gembeck MJ. Parent feeding restriction and child weight. The mediating role of child disinhibited eating and the moderating role of the parenting context. Appetite. 2009;52:726-34.

78. Hanson NI, Neumark-Sztainer D, Eisenberg ME, Story M, Wall M. Associations between parental report of the home food environment and adolescent intakes of fruits, vegetables and dairy foods. Public Health Nutr. 2005:8:77-85.

79. Wang Y, Beydoun MA, Li J, Liu Y, Moreno LA. Do children and their parents eat a similar diet? Resemblance in child and parental dietary intake: systematic review and meta-analysis. J Epidemiol Community Health. 2011;65:177-89.

80. Taylor CM, Wernimont SM, Northstone K, Emmett PM. Picky/fussy eating in children: review of definitions, assessment, prevalence and dietary intakes. Appetite. 2015;95:349-59.

81. Rodgers RF, Paxton SJ, Massey R, Campbell KJ, Wertheim EH, Skouteris H, Gibbons K. Maternal feeding practices predict weight gain and obesogenic eating behaviors in young children: a prospective study. Int J Behav Nutr Phys Act. 2013;10:24

82. Davison KK, Gicevic S, Aftosmes-Tobio A, Ganter C, Simon CL, Newlan S, Manganello JA. Fathers' representation in observational studies on parenting and childhood obesity: a systematic review and content analysis. Am J Public Health. 2016;106:e14-21.

83. Morgan PJ, Young MD, Lloyd AB, Wang ML, Eather N, Miller A, Murtagh EM, Barnes AT, Pagoto SL. Involvement of fathers in pediatric obesity treatment and prevention trials: a systematic review. Pediatrics. 2017;139:e20162635.

84. Moore DA, Goodwin TL, Brocklehurst PR, Armitage CJ, Glenny A-M. When are caregivers more likely to offer sugary drinks and snacks to infants? A qualitative thematic synthesis. Qual Health Res. 2017;27:74-88.

85. Li B, Adab P, Cheng KK. The role of grandparents in childhood obesity in Chinaevidence from a mixed methods study. Int J Behav Nutr Phys Act. 2015;12:91.

86. Gicevic S, Aftosmes-Tobio A, Manganello J, Ganter C, Simon C, Newlan S, Davison K. Parenting and childhood obesity research: a quantitative content analysis of published research 2009-2015. Obes Rev. 2016;17:724-34.

87. Taveras EM, Gillman MW, Kleinman KP, Rich-Edwards JW, Rifas-Shiman SL. Reducing racial/ethnic disparities in childhood obesity: the role of early life risk factors. JAMA Pediatr. 2013;167:731-8.

88. Caprio S, Daniels SR, Drewnowski A, Kaufman FR, Palinkas LA, Rosenbloom AL, Schwimmer JB, Kirkman MS. Influence of race, ethnicity, and culture on childhood obesity: implications for prevention and treatment. Obesity. 2008;16:2566-77.

89. Blaine RE, Fisher JO, Blake CE, Orloski A, Younginer N, Bruton Y, Ganter C, Rimm EB, Geller AC, Davison KK. Conditioned to eat while watching television? Low-income caregivers' perspectives on the role of snacking and television viewing among pre-schoolers. Public Health Nutr. 2016;19:1598-605.

90. Pescud M, Pettigrew S. Treats: Iow socioeconomic status Australian parents' provision of extra foods for their overweight or obese children. Health Promot J Austr. 2014;25:104-9. 
91. Wang D, van der Horst K, Jacquier E, Eldridge AL. Snacking among US children: patterns differ by time of day. J Nutr Educ Behav 2016,48. 369-375:e361.

92. Younginer NA, Blake CE, Davison KK, Blaine RE, Ganter C, Orloski A, Fisher JO. "what do you think of when I say the word 'snack'?" towards a cohesive definition among low-income caregivers of preschool-age children. Appetite. 2016;98:35-40.

93. Blake CE, Younginer, N., Fisher, J.O, Blaine, R.E, Orloski, A., Adler, R., Bruton, Y. P, Davison, K.K. : Child snacking contexts and purposes and their associated nutritional quality among low-income caregivers of preschoolers. In International Society of Behavioral Nutrition and Physical Activity (ISBNPA) Annual Meeting; San Diego, CA. 2014 of Conference: 431.

Submit your next manuscript to BioMed Central and we will help you at every step:

- We accept pre-submission inquiries

- Our selector tool helps you to find the most relevant journal

- We provide round the clock customer support

- Convenient online submission

- Thorough peer review

- Inclusion in PubMed and all major indexing services

- Maximum visibility for your research

Submit your manuscript at www.biomedcentral.com/submit
C) Biomed Central 